\title{
De novo transcriptome sequencing and analysis of male and female swimming crab (Portunus trituberculatus) reproductive systems during mating embrace (stage II)
}

Zhengfei Wang ${ }^{1}$, Linxia Sun ${ }^{1}$, Weibing Guan², Chunlin Zhou' ${ }^{1}$ Boping Tang ${ }^{1}$, Yongxu Cheng ${ }^{3}$, Jintian Huang ${ }^{4}$ and Fujun Xuan ${ }^{1,3^{*}}$ (D)

\begin{abstract}
Background: The swimming crab Portunus trituberculatus is one of the most commonly farmed crustaceans in China. As one of the most widely known and high-value edible crabs, it crab supports large crab fishery and aquaculture in China. Only large and sexually mature crabs can provide the greatest economic benefits, suggesting the considerable effect of reproductive system development on fishery. Studies are rarely conducted on the molecular regulatory mechanism underlying the development of the reproductive system during the mating embrace stage in this species. In this study, we used high-throughput sequencing to sequence all transcriptomes of the $P$. trituberculatus reproductive system.
\end{abstract}

Results: Transcriptome sequencing of the reproductive system produced 81,688,878 raw reads (38,801,152 and 42,887,726 reads from female and male crabs, respectively). Low-quality (quality <20) reads were trimmed and removed, leaving only high-quality reads (37,020,664 and 41,021,030 from female and male crabs, respectively). A total of 126,188 (female) and 164,616 (male) transcripts were then generated by de novo transcriptome assembly using Trinity. Functional annotation of the obtained unigenes revealed that a large number of key genes and some important pathways may participate in cell proliferation and signal transduction. On the basis of our transcriptome analyses and as confirmed by quantitative real-time $P C R$, a number of genes potentially involved in the regulation of gonadal development and reproduction of P. trituberculatus were identified: ADRA1B, BAP1, ARL3, and TRPA1.

Conclusion: This study is the first to report on the whole reproductive system transcriptome information in stage II of P. trituberculatus gonadal development and provides rich resources for further studies to elucidate the molecular basis of the development of reproductive systems and reproduction in crabs. The current study can be used to further investigate functional genomics in this species.

Keywords: Reproductive systems, Differentially expressed genes, Transcriptome, Portunus Trituberculatus

\footnotetext{
* Correspondence: swimming_crab@126.com

${ }^{1}$ Jiangsu Key Laboratory for Bioresources of Saline Soils, Jiangsu Synthetic

Innovation Center for Coastal Bio-agriculture, Jiangsu Provincial Key Laboratory of Coastal Wetland Bioresources and Environmental Protection, School of Ocean and Biological Engineering, Yancheng Teachers University, Yancheng 224001, Jiangsu Province, People's Republic of China

${ }^{3}$ Key Laboratory of Freshwater Aquatic Genetic Resources, Ministry of Agriculture, Shanghai Ocean University, Shanghai 200090, People's Republic of China

Full list of author information is available at the end of the article
} 


\section{Background}

The swimming crab (Portunus trituberculatus) is a commercially important crab species for both aquaculture and fisheries widely distributed in East Asian countries [1]. In China, this species spreads widely throughout marginal seas, including the East China Sea, South China Sea, Yellow Sea, and Bohai Sea [2]. P. trituberculatus is one of the most important marine species cultured in China because of its high nutritional content and economic value. This crab has a life cycle of 2-3 years and can reach sexual maturity in the first year $[3,4]$. Before the puberty molt, $P$. trituberculatus grows rapidly by frequent molting (every 5-30 d) [3]. Immediately after puberty molt and mating, the female $P$. trituberculatus usually starts vitellogenesis and ovarian development $[3,5]$. During these stages, the development of the reproductive system is vital to $P$. trituberculatus because not only are many larval crabs needed for aquaculture, an individual with a mature ovary is more widely known to consumers as well.

The reproductive system is fundamental to reproductive processes [6]. The reproductive system of the male crab contains paired testes, vas deferens, seminal vesicles, and a genital aperture, whereas the female crab consists of paired ovaries, oviducts, gonophores, and an external sperm reception area [7]. The development of the male and female reproductive system is crucial to the reproduction as well as commercial seed production of $P$. trituberculatus. The gonads (testes and ovaries) are the primary reproductive organs, and their development affects reproduction and sex differentiation [8]. Gonadal development consists of ovarian and testicular development and maturation, such as spermatogenesis, ovarian differentiation, and vitellogenesis. In male $P$. trituberculatus, spermatogenesis is mostly characterized by the differentiation of sperm cells and their maintenance before fertilization. In female $P$. trituberculatus, ovarian development is usually divided into 6 stages [5]. In stage I, the ovary is transparent and ribbonlike, and the main cells consist of oogonia and previtellogenic oocyte. In stage II (mating embrace), the ovary is milky white, and the main cells are composed of endogenous vitelloge and previtellogenic oocytes. In stage III, the ovary is buff and orange, and the main cell type is an exogenous vitellogenic oocyte. In stage IV, the main cell types are exogenous vitellogenic and nearlymature oocytes in the ovary, which turn into deep orange. In stage V, the ovary is ripe, and the main cell type is a mature oocyte. Finally, in stage VI, the crab has spawned, and the ovary turns light orange $[5,9,10]$.

The ovarian development of P.trituberculatus is initiated by copulation. When female pubertal molting and subsequent mating occur (stage II), the ovary remains milky white, and the main cell types are endogenous vitellogenic and previtellogenic oocytes $[5,9,10]$. Meanwhile, the special structure of the sperm plug is also formatted in the spermatheca during mating. In a mating embrace (stage II), seminal fluid proteins (including protease inhibitors, lectins, prohormones, peptides, and protective proteins such as antioxidants) are transferred to females with sperm $[9,11]$. Thus, to understand the regulatory mechanisms underlying reproductive development in this species during stage II, both whole reproductive systems should be considered together. The reason is that an increasing number studies show that male seminal material performs vital functions in female reproductive fitness, including ovarian development and sperm plug formation $[12,13]$. However, no studies identifying and characterizing stage II in P. trituberculatus have been conducted at the transcriptome level.

Transcriptome is a collection of transcripts within cells in certain physiological conditions [14], and transcripts can be used to examine the expression of genes at the RNA level. Transcriptome studies have rapidly emerged in recent years following the advent of next-generation sequencing technology, which provides high-throughput and low-cost sequencing that exhibits significant influence on genetic research. A number of studies have analyzed the gonad transcriptome and provided information about the molecular mechanisms underlying gonadal development and maturation [15-17]; but few have focused on the reproductive system. Various sequencing platforms have also been established, such as Solexa illumine, Roche 454, and ABI solid [18-20]. These technologies have been applied in studying the transcriptome of many aquatic animals, particularly in crustaceans [18-20].

In the present study, de novo assembly and highthroughput Illumina HiSeq sequencing were employed to obtain the comparative transcriptome of the female and male reproductive systems of $P$. trituberculatus during the mating embrace (stage II). Moreover, differentially expressed genes (DEGs) were identified and analyzed. The current study intends to quantify and identify spermatogenesis and ovarian differentiation related genes and identify the pathways involved. Findings from this transcriptome dataset can provide valuable resources to elucidate the molecular mechanisms underlying the physiological and morphological changes during stage II of the development of the whole reproductive system. Thus, the current study can provide valuable genomic information to understand the development of reproductive systems and the maturation of P. trituberculatus.

\section{Results}

Illumina sequencing and assembly

To obtain an overview of the reproductive system transcriptome of $P$. trituberculatus and identify the genes involved in the development and maturation of the 
reproductive systems, two cDNA libraries were prepared from pooled RNA extracts of female and male reproductive systems in stage II. These extracts were then sequenced using the Illumina Solexa platform. A total of $42,887,726$ and 38,801,152 raw reads were generated from male and female transcriptome sequencing, respectively. Ambiguous nucleotides and low-quality (quality <20) reads were trimmed and removed. The remaining high-quality reads, totaling to $41,021,030$ and 37,020,664 reads from male and female, respectively, were then obtained. All reads were submitted to the website of the National Center for Biotechnology Information Short Read Archive site, with accession number SRR5723730 and SRR5723731.

De novo transcriptome assembly with Trinity using reads from female and male crabs generated 126,188 and 164,616 transcripts, respectively. The average length of the transcripts in the female and male crabs were $954 \mathrm{bp}$ (ranging from $201 \mathrm{bp}$ to 20,074 bp) and $823 \mathrm{bp}$ (ranging from $201 \mathrm{bp}$ to 17,641 bp), respectively. The detailed sequencing and assembly results for the female and male crabs are shown in Table 1. We used Illumina sequencing to generate a substantial number of long sequences; the lengths of $17.25 \%(23,457)$ of the total transcripts exceeded $600 \mathrm{bp}$, and the lengths of $4.13 \%$ $(5,613)$ of the transcripts exceeded 1,000 bp (Fig. 1).

\section{Annotation of unique sequences}

Annotation of 88,804 unigenes was conducted using BLASTX [21] searches against the String, KEGG, Pfam, Swissprot, and non-redundant (NR) databases. The numbers of matched unigenes in the respective databases were as follows: 4021 (4.53\%), String database;

Table 1 Result of the de novo transcriptome assembly performed with Trinity

\begin{tabular}{llll}
\hline & Type & Unigene & Transcripts \\
\hline Female & Total sequence num & 70,807 & 126,188 \\
& Total sequence base & $50,955,454$ & $120,326,367$ \\
& Percent GC & 45.04 & 45.79 \\
& Largest & 20,074 & 20,074 \\
Smallest & 201 & 201 \\
Average & 719.64 & 953.55 \\
& N50 & 1268 & 1839 \\
& Total sequence num & 101,401 & 164,616 \\
& Total sequence base & $63,867,553$ & $135,502,734$ \\
& Percent GC & 46.30 & 46.58 \\
& Largest & 17,641 & 17,641 \\
& Smallest & 201 & 201 \\
& Average & 629.85 & 823.14 \\
& N50 & 934 & 1504 \\
\hline
\end{tabular}

8432 unigenes (9.50\%), KEGG; 10,924 unigenes (12.30\%), Pfam; 11,887 unigenes (13.39\%), Swissprot, and 17,558 unigenes (19.77\%), NR. The BLASTX top-hit species distribution of the 1928 annotated unigenes exhibited the highest homology to Zootermopsis nevadensis (11.34\%), followed by Daphnia pulex (1137, 6.69\%), Tribolium castaneum (657, 3.87\%), and Pediculus humanus (439, 2.58\%) (Fig. 2).

\section{Functional annotation of the transcriptome}

To functionally categorize the assembled unigenes, gene ontology (GO) assignment programs were used. The functions of 6046 annotated sequences were predicted. Blast2 GO was used to assign the GO terms. These unigenes were assigned to major GO categories (level 3), i.e., biological process, cellular component, and molecular function (Fig. 3). For biological processes, "cellular process" (54.00\%, GO: 0009987) was the most abundant term. The genes involved in the metabolic process (51.10\%, GO: 0008152), single-organism process (44.81\%, GO: 0044699), organic substance metabolic process (39.52\%, GO: 0071704), primary metabolic process (38.54\%, GO: 0044238), single-organism cellular process $(37.24 \%$, GO: 0044763), and cellular metabolic process (36.20\%, GO: 0044237) were also highly represented. For the cellular component, "cell" (30.67\%, GO: 0005623) and "cell part" (30.67\%, GO: 0044464) were the most represented categories, followed by "intracellular" (26.62\%, GO: 0005622), "intracellular part" (24.47\%, GO: 0044424), "membrane" (21.09\%, GO: 0016020), and "organelle" (19.90\%, GO: 0043226). For molecular function, "binding" ( $47.58 \%$, GO: 0005488) was the most prevalent, followed by "catalytic activity" (47.06\%, GO: 0003824), "organic cyclic compound binding" (28.59\%, GO: 0097159), "heterocyclic compound binding" (28.55\%, GO: 1,901,363), and "ion binding" (25.69\%, GO: 0043167) (Fig. 3). Among all GO terms, the biological process ontology comprised the largest proportion, followed by the molecular functions ontology and the cellular component ontology. Within the biological process ontology, 288 unigenes were identified as developmental process (GO: 0032502), 57 unigenes were annotated to reproduction (GO: 0000003), and 47 unigenes were annotated to growth (GO: 0040007).

To further analyze the possible pathways involved in the development of the $P$. trituberculatus reproductive system, all unigenes were mapped to the reference pathways in the KEGG database. A total of 8, 432 unigenes were mapped to 337 pathways representing biological systems involved in stage II of the development of the P. trituberculatus reproductive system. The most abundant pathways represented in the reproductive systems are the metabolic pathway (1, 112 unigenes, ko01100) and the biosynthesis of secondary metabolites (299 unigenes, ko01110) (Additional file 1: Table S1 and Fig. 4). Many unigenes were mapped to 


\section{Sequence length distribution}

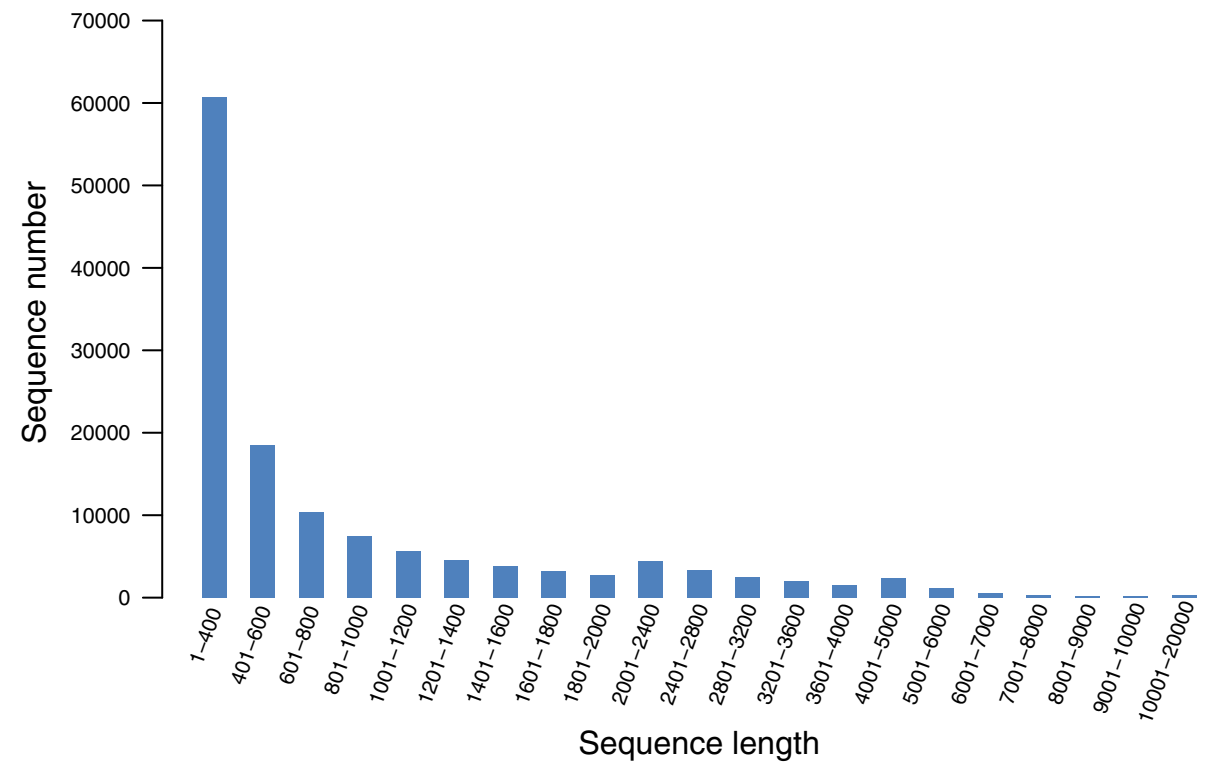

Fig. 1 Length distribution of the final assembled unigenes. The $X$ axis shows the sequence lengths of the unigenes, and the $Y$ axis shows the number of unigenes

pathways related to cell proliferation and signal transduction, including purine metabolism (185 unigenes, ko00230), the cAMP signaling pathway (171 unigenes, ko04024), the PI3K-Akt signaling pathway (163 unigenes, ko04151), regulation of the actin cytoskeleton (160 unigenes, ko04810), the Rap1 signaling pathway (152 unigenes, ko04015), RNA transport (151 unigenes, ko03013), protein processing in the endoplasmic reticulum (150 unigenes, ko04141), spliceosome (144 unigenes, ko03040), ribosome (133 unigenes, ko03010), lysosome (132 unigenes, ko04142), and ubiquitin-mediated proteolysis (131 unigenes, ko04120) (Additional file 1: Table S1 and Fig. 4).

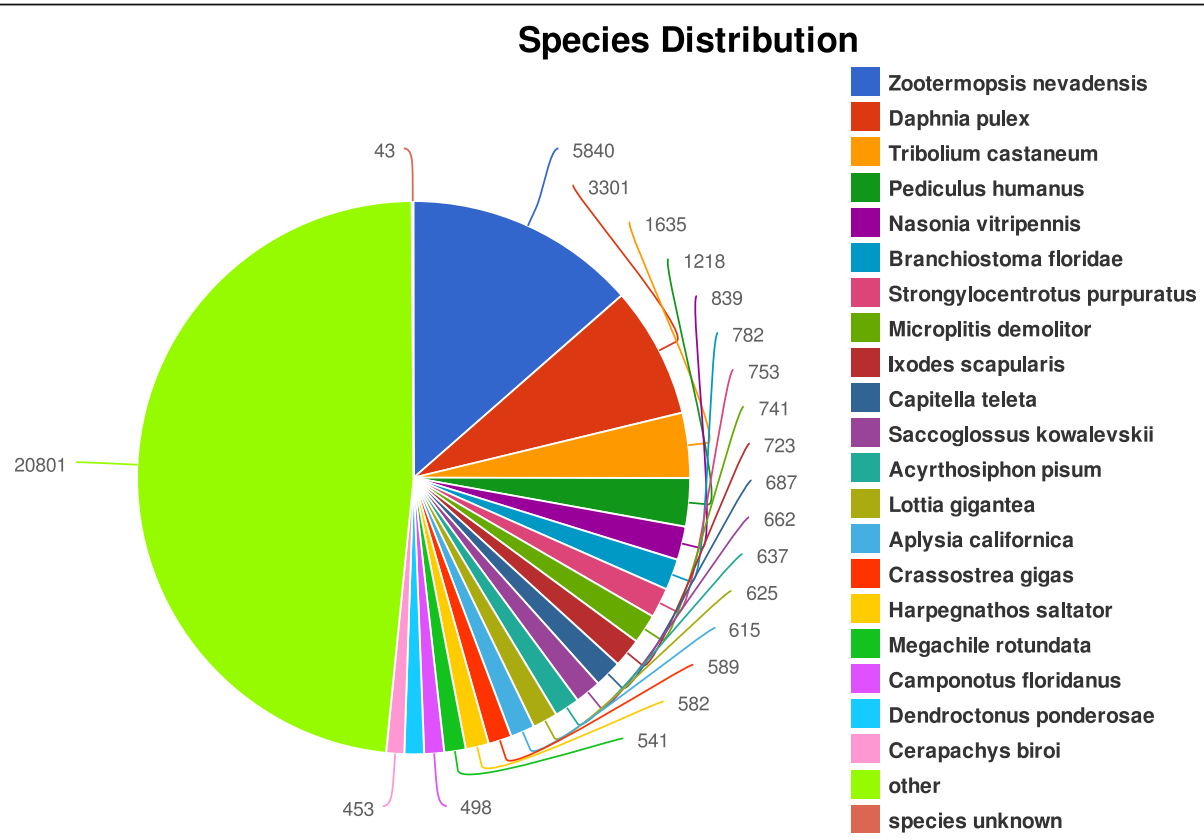

Fig. 2 Species distribution of BLASTx matches the reproductive system transcriptome unigenes. Each piece of fan indicates the number of top BLAST matches (the matches with the lowest E-value for each unigene) against the Genbank non-redundant (Nr) protein database for various species 


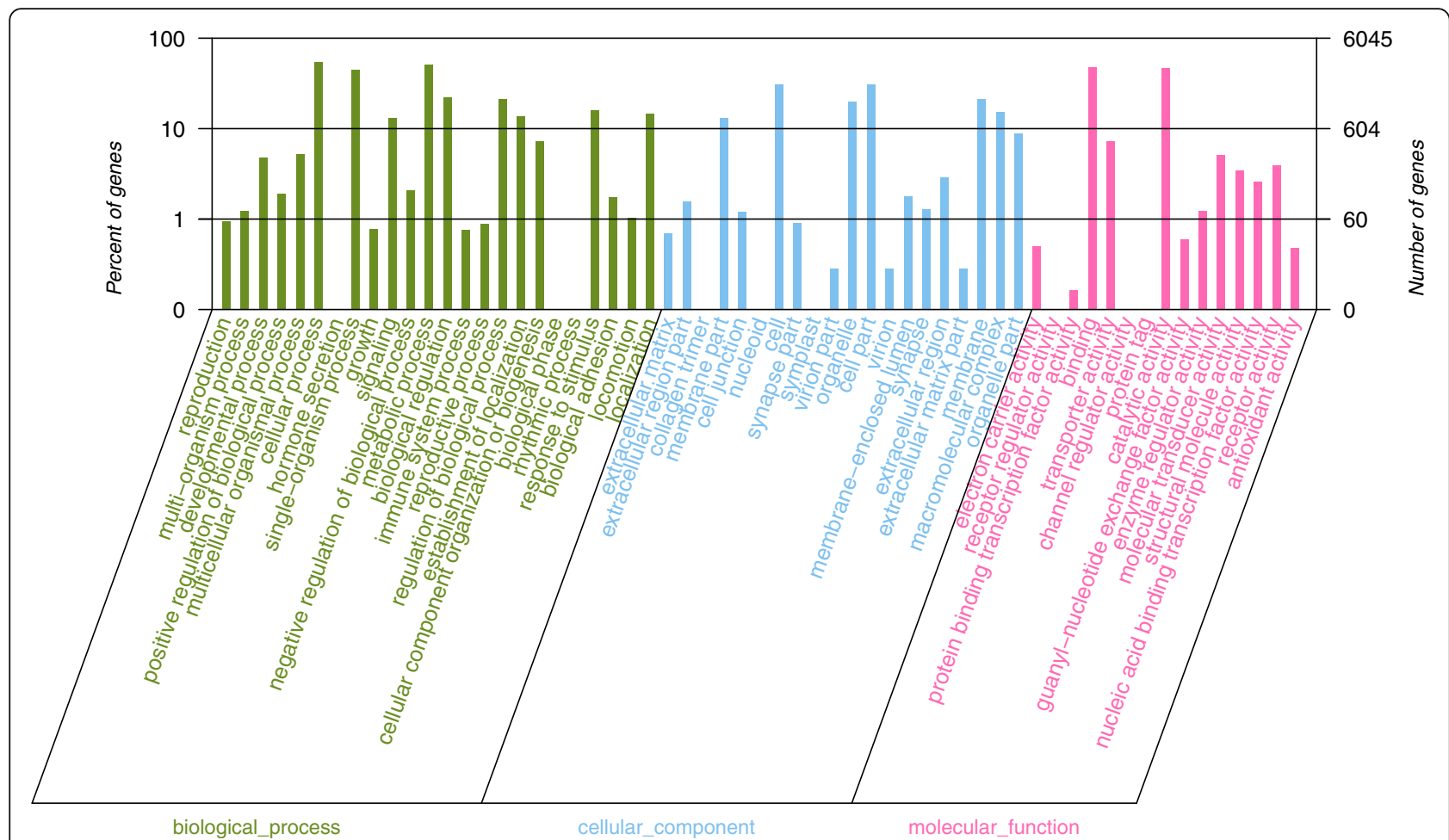

Fig. 3 Gene ontology $(\mathrm{GO})$ assignment of assembled unigenes of $P$. trituberculatus. $\mathrm{GO}$ terms were processed by Blast2Go and categorized at the 2nd level under 3 main categories (biological process, cellular component, and molecular function)

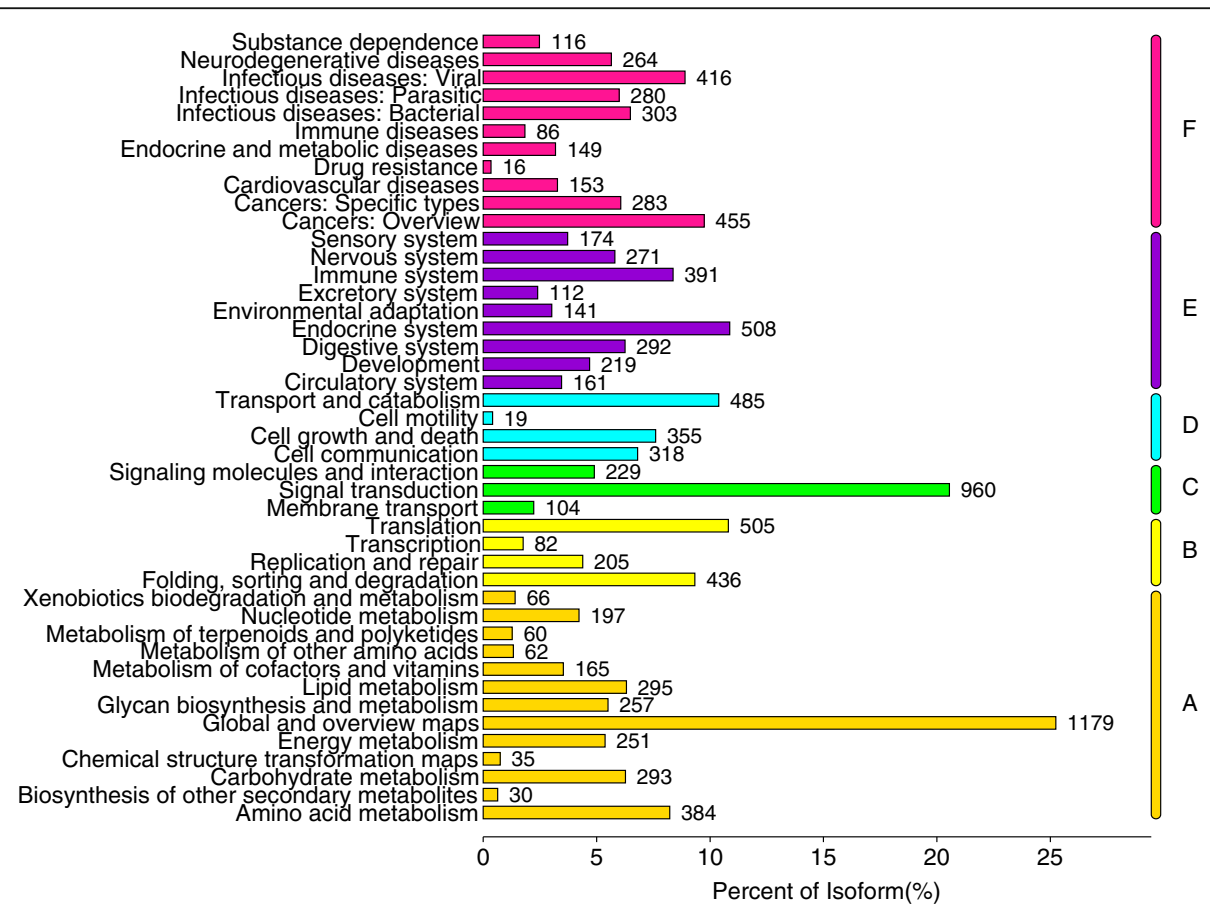

Fig. 4 Distribution of the mapped Kyoto Encyclopedia of Genes and Genomes (KEGG) pathways 
Analysis of differentially expressed genes between female and male reproductive systems

To identify and characterize the DEGs between the female and male in stage II, we found 9, 066 genes to be significantly differentially expressed (FDR $<=0.01$, $|\log \mathrm{FC}|>=1$ ) (Additional file 2: Table S2). Among these DEGs, 2, 723 were upregulated in the female and 6, 343 were downregulated (Additional file 3: Table S3). GO and KEGG analyses were also performed to understand the functions of these DEGs. These DEGs were then assigned to $37 \mathrm{GO}$ terms and 184 pathways through the KEGG database. DEGs were mostly enriched in molecular function and biological process, and many DEGs were related to gametogenesis and signal transduction, such as genes associated with transport, transporter activity, signal transducer activity, ion channel activity, and so on. Of the functional pathways, neuroactive ligandreceptor interaction and purine metabolism were most specifically expressed in $P$. trituberculatus. In addition, for specifically expressed genes in reproductive systems, several pathways were found associated with gonadal development and sex maintenance, such as homologous recombination, the Wnt signaling pathway, and progesterone-mediated oocyte maturation.

To validate the expression profiles obtained from Illumina sequencing analysis, 4 DEGs that play key roles in regulating gonadal differentiation and development were chosen for qRT-PCR analysis, using the same RNA samples. These DEGs include alpha-1B adrenergic receptor $(A D R A 1 B)$, BRCA1-associated protein 1 (BAP1), ADPribosylation factor-like $3(A R L 3)$, and transient receptor potential ankyrin 1 (TRPA1). Among these, the differential expression patterns of 3 genes are consistent with the results obtained by Illumina sequencing (Fig. 5). The qRTPCR results generally agreed with the deep sequencing

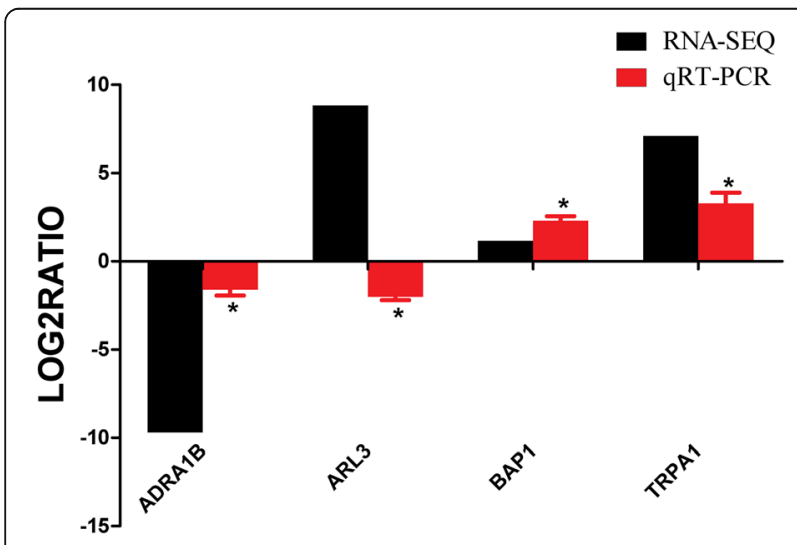

Fig. 5 qRT-PCR validation of differentially expressed genes analyzed by RNA-seq in female. qRT-PCR was performed for 4 genes that were identified as differentially expressed between the female and male reproductive systems. The $Y$ axis shows the relative mRNA expression levels. ${ }^{*} p<0.05$ analysis, which verified the data obtained by Illunima sequencing.

In addition, to study the regulatory mechanisms underlying the reproductive development of $P$. trituberculatus during the mating embrace (stage II), we set the immature crabs as the control group for expression analysis. Increased expression levels in the 4 genes (ADRA1B, BAP1, ARL3, and TRPA1) were detected in the male mature crabs, relative to those of immature crabs (Additional file 4: Figure S1). In the female mature crabs, the expression of the ARL3 gene was significantly decreased, whereas the expression levels of the $A D R A 1 B, B A P 1$, and TRPA1 genes increased (Additional file 5: Figure S2).

\section{Discussion}

Reproductive systems are important in the development of species and fulfill many pivotal functions, including gametogenesis, fertilization, and hormone secretion [7]. Numerous studies focused on gonad development and sexual differentially expressed genes and marker information; however, those study subjects were limited to gonad tissues (i.e., testis and ovary) [15-17]. In the present study, we performed de novo assembly of the transcriptome of the whole reproductive systems of female and male $P$. trituberculatus. Compared with those of separate gonad tissues, the transcriptomes of whole reproductive systems can offer more comprehensive information for research in gonadal development and reproduction.

The results of large-scale transcriptome sequencing of the swimming crab (the whole male and female reproductive systems) could provide resources for gene expression profiling studies as well as identification of functional classifications, molecular markers, and molecular pathways. In general, the high quality of long sequences enables us to gain more information about genes [15]. In the current study, 23,457 transcripts (17.25\%) had sequence lengths exceeding $600 \mathrm{bp}$, and 5613 transcripts $(4.13 \%)$ had sequence lengths exceeding $1000 \mathrm{bp}$. Therefore these datasets can provide a valuable resource for future studies on specific processes, functions, and pathways in the mating embrace (stage II) of P. trituberculatus.

The reproductive tissue development of crustaceans is deemed to be a dynamic process involving coordinated interactions between regulators that assemble or edit the cellular constituents supporting the developing gametes [17]. GO and KEGG analyses were used for gene function classification and annotation, and for obtaining background knowledge of gene functions that can help predict the role of protein interaction networks in cells [22]. We obtained GO and KEGG assignment results partially similar to the previous crab transcriptome 
sequencing; however, a large number of key genes and several important pathways may participate in cell proliferation and signal transduction (Figs. 3 and 4). Gametogenesis is a complex process involving cellular transformations that result in the production of male and female haploid germ cells, and it starts from the mitotic cell cycle followed by entry into meiosis and the completion of complex differentiation programs [23]. Signal transduction is the process by which a chemical or physical signal is transmitted through a cell as a series of molecular events, and this process is also critical for the timing and maintenance of normal gametogenesis [24]. In many arthropods, mating initiates a behavioral and physiological switch in females, triggering responses in several processes related to fertility [9]. During a mating embrace (stage II), seminal fluid proteins are transferred to the female species, inducing numerous physiological and behavioral post-mating changes in females [9]. Thus, gametogenesis and signal transduction could potentially be a primary precondition in stage II of P. trituberculatus.

$A D R A 1 B$, which targets the neuroactive-ligand receptor interaction pathway, is a member of the G proteincoupled receptor superfamily. This receptor activates phosphatidylinositol hydrolysis, which may be crucial in mitogenesis and regulates growth and proliferation in many cells [25]. Previous studies have shown that $A D R A 1 B$ controls male fertility, spermatogenesis, and the steroidogenic capacity of Leydig cells [26]. ADRA1B in adult golden hamsters affects the responsiveness of testicular steroidogenesis to catecholamines [27, 28]. These findings are consistent with our current findings. In our transcriptome analysis and qRT-PCR, $A D R A 1 B$ showed increased expression during stage II and higher transcripts in the male species than in the female species (Figs. 5 and Additional file 4: Figure S1), suggesting that this gene participates in the spermatogenesis and male fertilization of $P$. trituberculatus.

ADP-ribosylation factor-like 3 (ARL3), a member of the ADP-ribosylation factor family of GTP-binding proteins, can bind guanine nucleotides but lacks ADPribosylation factor activity [29]. In previous studies, the expression and function of $A R L 3$ during spermiogenesis were examined, and its potential importance in the regulation of male fertility and infertility was determined [30]. In mouse, $A R L 3$ was found to be expressed from birth, and the expression increased significantly when spermiogenesis began [31]. Thus, ARL3 as a novel manchette-related protein with an important role in the spermiogenesisand formation of sperm tail collar, was usually identified as one of the potential proteins involved in the initiation of spermatogenesis [30, 31]. In our qRT-PCR, ARL3 was significantly increased and highly expressed in the male crabs during stage II
(Figs. 5 and Additional file 4: Figure S1), suggesting that this protein can participate in the spermatogenesis of P. trituberculatus. Notably, a decrease in the expression of the ARL3 gene was detected in female mature crabs relative to the immature crabs (Additional file 5: Figure S2), suggesting that the $A R L 3$ gene played an important role in the reproductive system of male $P$. trituberculatus.

TRPA1 is a membrane-associated cation channelthat is widely expressed in neuronal cells and involved in nociception and neurogenic inflammation [32]. Normally, TRPA1 is activated and can cause an influx of cation ions, particularly $\mathrm{Ca}^{2+}$, into the activated cells; this increase in intracellular $\mathrm{Ca}^{2+}$ would trigger an action potential in neuronal cells [33]. TRPA1 was unexpectedly expressed in some non-neuronal cells, such as keratinocytes, synoviocytes, and gonads [34, 35]. To illustrate, TRPA1 is conserved between many invertebrates, except as a chemosensor for noxious compounds and as a sensor for temperature-driven behaviors [33, 36-38]. Given that high temperature can simulate growth and initiate early ovarian development [39], TRPA1 may be activated by temperature fluctuations in the ovary. In the present study, the expression of TRPA1 was higher in the ovaries than in the testes (Figs. 5 and Additional file 5: Figure S2), indicating the involvement of TRPA1 in the ovarian development of $P$. trituberculatus.

$B A P 1$ is an important nuclear ubiquitin hydrolase involved in the cell cycle regulation, cell proliferation, cellular differentiation, repair of DNA damage, and apoptosis [40]. Ubiquitin C-terminal hydrolase was found to be involved in sex differentiation in fish [41]. The function of this protein remains undetermined despite numerous studies conducted on ubiquitin hydrolases. Some researchers suggest that ubiquitin hydrolases play an important role in sperm acrosomal function and antipolyspermy defense during porcine fertilization [41]. The qRT-PCR result indicated that the expression of $B A P 1$ was increased in the stage II, and the expression level was significantly higher in the ovary $(p<0.05)$ than in the testes (Fig. 5 and Additional file 5: Figure S2). This preliminary result showed that BAP1 may play a crucial role in oogenesis and ovarian development. However, its physiological function needs further investigation.

\section{Conclusions}

A total of 135,992 transcripts and 88,804 unigenes were obtained among which were many genes potentially involved in gonadal development, gametogenesis, and signal transduction. Analysis of DEGs revealed 9066 significant genes between the female and male species in stage II, and 4 DEGs (ADRA1B, BAP1, ARL3, and TRPA1) were confirmed by qRT-PCR. This study is the first to report on the transcriptome of the reproductive system during a mating embrace (stage II) in P. trituberculatu. 
This study also provides abundant resources for further research on the molecular basis of reproduction and development in crabs. Future studies would certainly require evaluating the roles of the pathways involved and the gene expression profiles associated with those pathways.

\section{Methods}

\section{Sample preparation and RNA extraction}

A total of 10 pre-pubertal females (distinguished by the differences in the abdominal shape and the coloration of the second-to-last segment of the swimming appendage) [9] and 10 mature male crabs $(\mathrm{CW}>100 \mathrm{~mm}$, gonadal maturity) [42] were obtained in September 2006 (close to the mating peak) [43] from a local fishing port in Sheyang in Jiangsu Province. The crabs are transferred to the laboratory in Jiangsu key laboratory for bioresources of saline soils at Yancheng Teachers University and then pairs of crabs ( 1 male and 1 female) were randomly grouped and reared in ten $200-\mathrm{l}$ aquaria at $25^{\circ} \mathrm{C}$ and salinity of 30 for mating observations. When prepubertal females (Molt cycle D3-D4) initiated molting the pre-copulatory embrace was formatted by positioning females underneath males (stage II) [10]. Prior to copulation, the paired crabs were placed in an ice bath until anesthetized, and the whole male and female reproductive systems were dissected, frozen in liquid nitrogen and stored at $-80{ }^{\circ} \mathrm{C}$. Total RNA was then extracted from the whole male and female reproductive system with TRIzol Reagent (Invitrogen) in accordance with the instructions of the manufacturer and then treated with DNase I. Subsequently, cDNA libraries were generated with the TruseqTM RNA sample prep kit, and indexcoded samples were clustered with cBot Truseq PE Cluster Kit v3-cBot-HS. Finally, the male and female libraries were sequenced on Hiseq2000 TruSeq SBS Kit v3-HS.

\section{Date analysis of de novo sequencing data}

To obtain high-quality clean data, raw data were first filtered by removing the reads containing low-quality reads $(<20)$ and poly-N reads (> 20\% reads) in SeqPrep (https://github.com/jstjohn/SeqPrep) and Sickle (https:// github.com/najoshi/sickle). De novo transcriptome assembly was accomplished using Trinity (http://trinityr naseq.sourceforge.net/) $[44,45]$. The gene functions of all assembled unigenes were annotated based on the following databases with NR protein sequences, including Swissprot (a manually annotated and reviewed protein sequence database); Protein Information Resource (PIR); Protein Data Bank (PDB). Unigenes annotatation and characteristics of homology search of unigenes against the NR database. Similarly, we obtained GO annotations by Blast2Go (http://www.blast2go.com/b2ghome) $[46,47]$. KEGG pathway annotation and KEGG orthology assignments were obtained with the KEGG Automatic Annotation Server [48].

\section{Analysis of differentially expressed genes}

According to the results of all sample and reference genome alignments, the number of reads per kilo-base of exon model per Million (RPKM) mapped reads [49] by RSEM (http://deweylab.biostat.wisc.edu/rsem/) [50] and EdgeR (http://www.bioconductor.org/packages/2.12/bioc/ html/edgeR.html) [51] were calculated to obtain the differential expression of genes. By the standard FDR $<=0.01$, $|\log F C|>=1$, significant differentially expressed genes were selected.

\section{Quantitative real-time PCR analysis}

We chose 4 genes that were differentially expressed in the reproductive system for qRT-PCR verification. In addition, on the basis of the KEGG or GO function, these particular genes were selected to verify whether to conform to the transcriptome data. To compare the gene expression of the immature crabs with that of the mature crabs, we set the prepubertal crabs as the control group. The genespecific primers were designed by Primer Premier 6 (Table 2). PCR reaction was run on the Applied Biosystem 7500 real-time PCR system, using $2 \times$ SYBR Green qPCR Mix as recommended by Aidlab Biotechnologies. The reaction mixtures $(25 \mu \mathrm{L})$ contained $12.5 \mu \mathrm{L}$ of $2 \times$ SYBR qPCR Mix, $1 \mu \mathrm{L}$ of cDNA, $1 \mu \mathrm{L}$ of forward and reverse primers, and $10.5 \mu \mathrm{L}$ of RNase-free $\mathrm{H}_{2} \mathrm{O}$. PCR was performed at $95{ }^{\circ} \mathrm{C}$ for $3 \mathrm{~min}, 40$ cycles of $95{ }^{\circ} \mathrm{C}$ for $15 \mathrm{~s}, 60{ }^{\circ} \mathrm{C}$ for $15 \mathrm{~s}$, and $72{ }^{\circ} \mathrm{C}$ for $25 \mathrm{~s}$. At the end of the reaction, a melting curve was generated. PCR was conducted in 3technological replicates and 2 control groups by using all target genes and $\beta$-actin control gene [52]. The data for the 2 samples were calculated as the mean of the relative quality value such that the Cycle Threshold (CT) mean of each gene must be less than 30 . Finally, relative gene expression was analyzed using the $2^{-\Delta \Delta C T}$ method $[53,54]$.

\begin{tabular}{|c|c|c|}
\hline $\begin{array}{l}\text { Gene } \\
\text { Name }\end{array}$ & Forward primer sequence $\left(5^{\prime}-3^{\prime}\right)$ & Reverse primer sequence $\left(5^{\prime}-3^{\prime}\right)$ \\
\hline$\beta$-actin & ACTGGGACGACATGGAGAAGATC & AAACCTTACCACTCCCGCC \\
\hline ADRA1B & TCTGGTCGCTGTGCGTGAT & ATCAAGACCACCAAGACATCCG \\
\hline$A R L 3$ & CATCCCTTGCTCTACATTACTTCC & AAACGACGGGTGCCACAG \\
\hline BAP1 & ACCCGCACTCCTCCCTTAT & GAGAACCAAGTGGAGCAGACA \\
\hline TRPA1 & AGTAGCGGCAACATGTCCACC & CCAAACTAACCCTGAAAGACCG \\
\hline
\end{tabular}




\section{Additional files}

Additional file 1: Table S1. Unigenes were mapped to the reference canonical pathways in KEGG. (XLS 1156 kb)

Additional file 2: Table S2. Differentially expressed genes (DEGs) between the ovary and testis. (XLS $1611 \mathrm{~kb}$ )

Additional file 3: Table S3. Different up-regulated and down-regulated genes in reproductive system. (XLS $13119 \mathrm{~kb}$ )

Additional file 4: Figure S1. qRT-PCR was performed for 4 genes that were identified as differentially expressed between the immature and mature male reproductive systems. The $Y$ axis shows the relative mRNA expression levels. ${ }^{*} p<0.05 * * 0<0.01$. (PDF $1694 \mathrm{~kb}$ )

Additional file 5: Figure S2. qRT-PCR was performed for 4 genes that were identified as differentially expressed between the immature and mature female reproductive systems. The $Y$ axis shows the relative mRNA expression levels. ${ }^{*} p<0.05{ }^{* *} p<0.01$. (PDF $1820 \mathrm{~kb}$ )

\section{Abbreviations}

DEGs: Differentially expressed genes; EN: Endogenous vitellogeic oocyte; EX: Xogenous vitellogenic oocyte; GO: Gene Ontology; KEGG: Kyoto Encyclopedia of Genes and Genomes; MO: Mature oocyte; NCBI: National Center for Biotechnology Information; NO: Nearly-mature oocyte; PDB: Protein data bank: PIR: Protein Information Resource; PR: Previtellogenic oocyte; SNPs: Single nucleotide polymorphisms; SRA: Short Read Archive; SSRs: Simple sequence repeats

\section{Acknowledgements}

The authors thank the Shanghai Major Biotechnology Corporation for the assistance with data processing and bioinformatic analysis. We are indebted to Ming Lei and Lingjiang He for support and discussions.

\section{Funding}

This study was supported by grants from National Natural Science Foundation of China to FJX (No. 41606149), National Natural Science Foundation of China to ZFW (No. 31702014), Postdoctoral Science Foundation of China to FJX (No. 2015 M571545), Jiangsu Provincial Natural Science Foundation of China to FJX (No. BK20140461), Jiangsu Provincial Key Laboratory for Bioresources of Saline Soils Open Foundation to ZFW (Grant no. JKLBS2016007), and Doctoral Scientific Research Foundation of Yancheng Teachers University to ZFW.

\section{Availability of data and materials}

The data supporting the results of this article are included within the article and in its additional files.

\section{Authors' contributions}

FJX, ZFW and LXS designed and conceived the experiment. WBG and BPT collected and processed the samples. ZFW, CLZ and FJX performed the data analysis and draft the manuscript. YXC and JTH helped to improve the manuscript. All authors read and approved the final manuscript.

\section{Ethics approval and consent to participate}

Not applicable. The sampling location was not privately-owned or protected, and field sampling did not involve protected species.

\section{Consent for publication}

Not applicable.

\section{Competing interests}

I confirm that I have read BioMed Central's guidance on competing interests and have included a statement in the manuscript on any competing interests.

The authors declare that they have no competing interests.

\section{Publisher's Note}

Springer Nature remains neutral with regard to jurisdictional claims in published maps and institutional affiliations.

\section{Author details}

Jiangsu Key Laboratory for Bioresources of Saline Soils, Jiangsu Synthetic Innovation Center for Coastal Bio-agriculture, Jiangsu Provincial Key Laboratory of Coastal Wetland Bioresources and Environmental Protection, School of Ocean and Biological Engineering, Yancheng Teachers University, Yancheng 224001, Jiangsu Province, People's Republic of China. ${ }^{2}$ Key Laboratory of Shanghai Education Commission for Oceanic Fisheries Resources Exploitation, Shanghai Ocean University, Shanghai 200090, People's Republic of China. ${ }^{3}$ Key Laboratory of Freshwater Aquatic Genetic Resources, Ministry of Agriculture, Shanghai Ocean University, Shanghai 200090, People's Republic of China.

${ }^{4}$ Yancheng Institute of Technology, Yancheng 224051, People's Republic of China.

Received: 26 May 2017 Accepted: 20 December 2017

Published online: 03 January 2018

\section{References}

1. Wu X, Cheng Y, Zeng C, Wang C, Yang X. Reproductive performance and offspring quality of wild-caught and pond-reared swimming crab Portunus trituberculatus broodstock. Aquaculture. 2010:301(1-4):78-84.

2. Liu Y, Liu R, Ye L, Liang J, Xuan F, Genetic XQ. Differentiation between populations of swimming crab Portunus trituberculatus, along the coastal waters of the East China Sea. Hydrobiologia. 2009;618(1):125-37.

3. Wang W, Wu X, Liu Z, Zheng H, Cheng Y. Insights into Hepatopancreatic functions for nutrition metabolism and ovarian development in the crab Portunus trituberculatus: gene discovery in the comparative transcriptome of different Hepatopancreas stages. PLoS One. 2014;9(1):e84921.

4. Song HT, Ding YP, Population XYJ. Component characteristics and migration distribution of the Portunus Trituberculatus in the coast water of Zhejiang Province. Mater. Sci Bull. 1989:8:66-74.

5. XG W, Yao GG, Yang XZ, Cheng YX, Wang CLA. Study on the ovarian development of Portunus trituberculatus in East China Sea during the first reproductive cycle. Acta Oceanol Sin. 2007;29(4):121-7.

6. Vallina M, Moyano MPS, Cuartas El, Vallina M, Gavio MA. Reproductive system and size maturity of the paddle crab Ovalipes trimaculatus (Brachyura: Portunidae) along the argentine coast. J Crustacean Biol. 2014;34(3):357-66.

7. Nagaraju GPC. Reproductive regulators in decapod crustaceans: an overview. J Exp Biol. 2011;214(1):3-16.

8. Lu J, Luan P, Zhang X, Xue S, Peng L, Mahbooband S, et al. Gonadal transcriptomic analysis of yellow catfish (Pelteobagrus fulvidraco): identification of sex-related genes and genetic markers. Physiol Genomics. 2014; 46(21):798-807.

9. Xuan F, Bao C, Tang F, Tang B, Zhou C. Reproductive molt and mating behavior of the swimming crab Portunus trituberculatus in the laboratory-reard condition. Chin. J Zool. 2014:49:579-86.

10. Xuan FJ, Guan WB, Dai XJ, Zhu JF. Structure and content of spermathecae of Portunus trituberculatus from East China Sea. Chin. J Zool. 2009:44:1-11.

11. Polak M, Tstarmer W, Barker JSFA. Mating plug and male mate choice inDrosophila hibisciBock. Anim Behav. 1998;56(4):919-26.

12. Avila FW, Sirot LK, LaFlamme BA, Rubinstein CD, Wolfner MF. Insect seminal fluidproteins: identification and function. Annu Rev Entomol. 2011;56:21-40.

13. Mangels R, Tsung K, Kwan K, Dean MD. Copulatory plugs inhibit the reproductive success of rival males. J Evol Biol. 2016;11(29):2289-96.

14. Fan $Y$, LiHua $H$, AiBing $Z$. High-throughput transcriptome sequencing technology and its applications in Lepidoptera. Acta Entomol Sin. 2014; 57(8):991-1000

15. Meng X, Liu P, Jia F, Li J, Gao BQ. De novo transcriptome analysis of Portunus trituberculatus ovary and testis by RNA-Seq: identification of genes involved in gonadal development. PLoS One. 2015;10(6):e0128659.

16. Gao J, Wang X, Zou Z, Jia X, Wang Y, Zhang Z. Transcriptome analysis of the differences in gene expression between testis and ovary in green mud crab (Scylla paramamosain). BMC Genomics. 2014;15(1):585.

17. Yang Y, Wang J, Han T, Liu T, Wang C, Xiao J, et al. Ovarian transcriptome analysis of Portunus trituberculatus provides insights into genes expressed during phase III and IV development. PLoS One. 2015;10(10):e0138862.

18. LV J, Liu P, Wang Y, Gao B, Chen P, Li J. Transcriptome analysis of Portunus trituberculatus in response to salinity stress provides insights into the molecular basis of osmoregulation. PLoS One. 2013;8(12):e82155

19. Cornman RS, Bennett AK, Murray KD, Evans JD, Elsik CG, Aronstein K Transcriptome analysis of the honey bee fungal pathogen, Ascosphaera apis: implications for host pathogenesis. BMC Genomics. 2012:13(1):285.

20. Calabrese C, Mangiulli M, Manzari C, Paluscio AM, Caratozzolo MF, Marzano F, et al. A platform independent RNA-Seq protocol for the detection of transcriptome complexity. BMC Genomics. 2013;14(1):855. 
21. Camacho C, Coulouris G, Avagyan V, Ma N, Papadopoulos J, Bealer K, et al. BLAST+: architecture and applications. BMC bioinformatics. 2009;10(1):421.

22. Liu Y, Hui M, Cui Z, Luo D, Song C, Li Y, et al. Comparative transcriptome analysis reveals sex-biased gene expression in juvenile Chinese mitten crab Eriocheir sinensis. PLoS One. 2015;10(7):e0133068.

23. Li Q, Wang Y-L, Xie J, Sun W-J, Zhu M, He L. Characterization and expression of DDX6 during gametogenesis in the Chinese mitten crab Eriocheir sinensis. Genet Mol Res. 2015;14(2):4420-37.

24. Hermann BP, Heckert LL. Transcriptional regulation of the FSH receptor: new perspectives. Mol Cell Endocrinol. 2007;260-262(1):100-8.

25. Allen LF, Lefkowitz RJ, Caron MG, Cotecchia S. G-protein-coupled receptor genes as protooncogenes: constitutively activating mutation of the alpha $1 \mathrm{~B}$-adrenergic receptor enhances mitogenesis and tumorigenicity. Proc Natl Acad Sci. 1991;88(24):11354-8.

26. Mhaoutykodja S, Lozach A, Habert R, Tanneux M, Guigon C, Brailly-Tabard S, et al. Fertility and spermatogenesis are altered in alpha1b-adrenergic receptor knockout male mice. J Endocrinol. 2007:195(2):92-281.

27. Mu F, Jing Y, Qin N, Zhu H-Y, Liu D, HYuan S-G. Novel polymorphisms of ADRA1B and PPARGC1B genes and their association with egg production traits in local Chinese Dagu hens. Asian-Australas J Anim Sci. 2015;29(9): 1256-64.

28. Mayerhofer A, Bartke A, Steger RW. Catecholamine effects on testicular testosterone production in the gonadally active and the gonadally regressed adult golden hamster. Biol Reprod. 1989;40(4):752-61.

29. Schrick JJ, Vogel P, Abuin A, Hampton B, Rice DS. ADP-ribosylation factorlike 3 is involved in kidney and photoreceptor development. Am J Pathol. 2006;168(4):1288-98.

30. Huang $X-Y$, Sha J-H. Proteomics of spermatogenesis: from protein lists to understanding the regulation of male fertility and infertility. Asian J Androl. 2011;13(1):18-23.

31. Qi Y, Jiang M, Yuan $Y, B i$, Z Zheng B, Guo X, et al. ADP-ribosylation factor-like 3, a manchette-associated protein, is essential for mouse spermiogenesis. Mol Hum Reprod. 2013;19(5):327-35.

32. Peng G, Kashio M, Li T, Dong X, Tominaga M, Kadowaki T. TRPA1 channels inDrosophilaand honey bee Ectoparasitic mites share heat sensitivity and temperature-related physiological functions. Front Physiol. 2016;7(447):1-10.

33. Nummenmaa E, Hämäläinen M, Moilanen LJ, Paukkeri EL, Nieminen RM, Moilanen $\mathrm{T}$, et al. Transient receptor potential ankyrin 1 (TRPA1) is functionally expressed in primary human osteoarthritic chondrocytes. Arthritis Res Ther. 2016:18(1):185-96.

34. Anand U, Otto WR, Facer P, Zebda N, Selmer I, Gunthorpe MJ, et al. TRPA1 receptor localisation in the human peripheral nervous system and functional studies in cultured human and rat sensory neurons. Neurosci Lett. 2008;438(2):221-7.

35. Hatano N, Itoh Y, Suzuki H, Muraki Y, Hayashi H, Onozaki K, et al. Hypoxiainducible factor-1a (HIF1a) switches on transient receptor potential ankyrin repeat 1 (TRPA1) gene expression via a hypoxia response element-like motif to modulate cytokine release. J Biol Chem. 2012;287(38):31962-72.

36. Bautista DM, Movahed P, Hinman A, Axelsson HE, Sterner O, Hogestatt ED, et al. Pungent products from garlic activate the sensory ion channel TRPA1. Proc Natl Acad Sci U S A. 2005;102(34):12248-52

37. Bandell M, Story GM, Hwang SW, Viswanath V, Eid SR, Petrus MJ, et al. Noxious cold ion channel TRPA 1 is activated by pungent compounds and bradykinin. Neuron. 2004;41(6):849-57.

38. Jordt SE, Bautista DM, Chuang HH, McKemy DD, Zygmunt PM, Hogestatt ED, et al. Mustard oils and cannabinoids excite sensory nerve fibres through the TRP channel ANKTM1. Nature. 2004:427(6971):260-5.

39. Kinne O. Temperature: animals - invertebrates. Mar Ecol. 1970;1:407-514.

40. Owen D, Sheffield BS, lonescu D, Churg A. Loss of BRCA1-associated protein 1 (BAP1) expression is rare in non-small cell lung cancer. Hum Pathol. 2016; 60:82-5.

41. Fujiwara Y, Hatano K, Hirabayashi T, Miyazaki Jl. Ubiquitin C-terminal hydrolase as a putative factor involved in sex differentiation of fish (temperate wrasse, Halichoeres poecilopterus). Comput Phys Commun. 1994;12(2):13-20.

42. Xuan F, Bao C, Tang F, Tang B, Zhou C. Current fishing practices may induce low risk of sperm limitation in female swimming crab Portunus trituberculatus in the East China Sea. Aquat Biol. 2014;20:45-153.

43. Mu CK, Song WW, Li RH, Chen Y, Hao GJ, Wang CL. Identification of differentially expressed proteins relating to ovary development in Portunus trituberculatus. Aquaculture. 2014:426-427:148-53.
44. Grabherr MG, Haas BJ, Yassour M, Levin JZ, Thompson DA, Amit I, et al. Full-length transcriptome assembly from RNA-Seq data without a reference genome. Nat Biotechnol. 2011;29(7):644-52.

45. Haas BJ, Papanicolaou A, Yassour M, Grabherr M, Blood PD, Bowden J, et al. De novo transcript sequence reconstruction from RNA-seq using the trinity platform for reference generation and analysis. Nat Protoc. 2013;8(8):1494-512.

46. Conesa A, Götz S, García-Gómez JM, Terol J, Talón M, Robles M. Blast2GO: a universal tool for annotation, visualization and analysis in functional genomics research. Bioinformatics. 2005:21(18):3674-6.

47. Ye J, Fang L, Zheng H, Zhang Y, Chen J, Zhang Z, et al. WEGO: a web tool for plotting GO annotations. Nucleic Acids Res. 2006:34:293-7.

48. Kanehisa M, Goto SKEGG. kyoto encyclopedia of genes and genomes. Nucleic Acids Res. 2000;28(1):27-30.

49. Palmasilva C, Ferro M, Bacci M, Turchettozolet AFPKM. Values for each transcriptome. Mol Ecol Resour. 2015;16(4):105-16.

50. Zhao Q, Hautamaki V, Franti PRSEM. An accelerated algorithm on repeated EM. Sixth international conference on image and graphics. IEEE. 2011;1:135-40.

51. Dai Z, Sheridan JM, Gearing LJ, Moore DL, Su S, Wormald S, et al. edgeR: a versatile tool for the analysis of shRNA-seq and CRISPR-Cas9 genetic screens. F1000 Research. 2014;3:95.

52. Pfaffl MW. A new mathematical model for relative quantification in real-time RT_PCR. Nucleic Acids Res. 2001;29(9):e45.

53. Oddmund $\mathrm{N}$, Jan TK, Ragne KF, Reino H. Error propagation in relative real-time reverse transcription polymerase chain reaction quantification models: the balance between accuracy and precision. Anal Biochem. 2006:356(2):182-93.

54. Cantsilieris S, Baird PN, White SJ. Molecular methods for genotyping complex copy number polymorphisms. Genomics. 2012;101(2):86-93.

\section{Submit your next manuscript to BioMed Central and we will help you at every step:}

- We accept pre-submission inquiries

- Our selector tool helps you to find the most relevant journal

- We provide round the clock customer support

- Convenient online submission

- Thorough peer review

- Inclusion in PubMed and all major indexing services

- Maximum visibility for your research

Submit your manuscript at www.biomedcentral.com/submit 